\title{
Quaternion-valued single-phase model for three-phase power system
}

\author{
Xiaoming Gou*, Zhiwen Liu*, Wei Liu ${ }^{* *}$, Yougen Xu* , Jiabin Wang**
}

\begin{abstract}
In this work, a quaternion-valued model is proposed in lieu of the Clarke's $\alpha, \beta$ transformation to convert three-phase quantities to a hypercomplex single-phase signal. The concatenated signal can be used for harmonic distortion detection in three-phase power systems. In particular, the proposed model maps all the harmonic frequencies into frequencies in the quaternion domain, while the Clarke's transformation-based methods will fail to detect the zero sequence voltages. Based on the quaternion-valued model, the Fourier transform, the minimum variance distortionless response (MVDR) algorithm and the multiple signal classification (MUSIC) algorithm are presented as examples to detect harmonic distortion. Simulations are provided to demonstrate the potentials of this new modeling method.
\end{abstract}

K e y w o r ds: harmonics detection, Fourier transform, minimum variance distortionless response, multiple signal classification, quaternion, three-phase power system

\section{Introduction}

Power quality control is one of the major concerns for power delivery systems to function reliably, and it requires measurements of voltage characteristics, among which the frequency measurement is a non-trivial task due to the presence of voltage sags and voltage harmonics mostly caused by nonlinear loads [1]. In the particular case of three-phase power systems, the Clarke's $\alpha, \beta$ transformation is widely used as the preprocessing method to create a complex-valued single-phase signal from the real-valued three-phase signals [2], so that traditional complex-valued spectrum estimation methods can be applied, such as the MVDR method or the recently proposed Iterative MVDR (I-MVDR) method [3,4]. To improve the resolution, we can further apply the subspace methods and one representative example is the MUSIC method [5].

However, all the zero sequence voltages will be cancelled out in the complex-valued signal and hence can not be detected. Although these harmonic voltages would simply be blocked by a delta transformer, they will add up in the neutral, leading to overheating in the transformer and potential fire hazards [6]. To detect these harmonics, as well as harmonics of other orders, we propose a quaternion-valued model and all the traditional spectrum estimation methods can be extended to this domain, such as MVDR and MUSIC. We will show that harmonics of all orders will be reserved in the resulting quaternion-valued signal and will be detected by relevant estimation methods. This quaternion-valued model has been previously presented for the tracking of the fundamental frequency in the presence of voltage sags using the Kalman filter algorithm [7]. In this paper, we demonstrate a complementary merit of this model when dealing with harmonic voltages.

\section{Complex-valued frequency estimation for three-phase power systems}

\subsection{A brief review}

We consider the following discrete-time balanced threephase power system in the presence of $H-1$ harmonic distortions

$$
\begin{aligned}
& v_{a}(n)=\sum_{h=1}^{H} V_{h} \cos \left(h\left(\Omega n T_{s}+\phi\right)\right)+\varepsilon_{a}(n), \\
& v_{b}(n)=\sum_{h=1}^{H} V_{h} \cos \left(h\left(\Omega n T_{s}+\phi-\frac{2 \pi}{3}\right)\right)+\varepsilon_{b}(n), \\
& v_{c}(n)=\sum_{h=1}^{H} V_{h} \cos \left(h\left(\Omega n T_{s}+\phi+\frac{2 \pi}{3}\right)\right)+\varepsilon_{c}(n)
\end{aligned}
$$

where $\left\{V_{h}\right\}_{h=1}^{H}$ are the amplitudes of the harmonic signals, $\Omega$ is the fundamental (angular) frequency, $T_{s}$ is the sampling interval, $\phi$ is the signal phase, and $\varepsilon_{a}(n), \varepsilon_{b}(n), \varepsilon_{c}(n)$ are the measurement noise.

Traditionally, the three-phase signals will be converted to a complex-valued single-phase signal via the Clarke's $\alpha, \beta$ transformation. Firstly, the three-phase signals are mixed into two parts, namely $v_{\alpha}(n)$ and $v_{\beta}(n)$, where

$$
\left[\begin{array}{l}
v_{\alpha}(n) \\
v_{\beta}(n)
\end{array}\right]=\mathbf{T}\left[\begin{array}{l}
v_{a}(n) \\
v_{b}(n) \\
v_{c}(n)
\end{array}\right],
$$

* School of Information and Electronics Beijing Institute of Technology, Beijing 100081, China, xmgou@bit.edu.cn, zwliu@bit.edu.cn, yougenxu@bit.edu.cn, ** Department of Electronic and Electrical Engineering University of Sheffield, Sheffield S1 3JD, United Kingdom, w.liu@sheffield.ac.uk, j.b.wang@sheffield.ac.uk 


$$
\mathbf{T}=\frac{2}{3}\left[\begin{array}{ccc}
1 & -\frac{1}{2} & -\frac{1}{2} \\
0 & \frac{\sqrt{3}}{2} & -\frac{\sqrt{3}}{2}
\end{array}\right] .
$$

Then these two parts will be merged as a complex-valued signal $v_{\mathrm{cv}}(n)=v_{\alpha}(n)+i v_{\beta}(n)$.

With this complex-valued signal, we can exploit the MVDR spectrum to locate the frequencies, and it is given by

$$
S_{\mathrm{MVDR}}(\Omega)=\frac{1}{s^{\mathrm{H}}(\Omega) \mathbf{R}^{-1} \mathbf{s}(\Omega)}
$$

where $(\cdot)^{\mathrm{H}}$ is the Hermitian-transpose operation, $\mathbf{R}$ is the covariance matrix of dimension $M \times M$, and

$$
\mathbf{s}(\Omega)=\left[1, \mathrm{e}^{i \Omega T_{s}}, \ldots, \mathrm{e}^{-i \Omega T_{a}(M-1)}\right]^{\top}
$$

is the frequency sweeping vector.

We can also use the MUSIC spectrum which is expressed as

$$
S_{\text {MUSIC }}(\Omega)=\frac{1}{\left\|\mathbf{s}^{\mathrm{H}}(\Omega) \mathbf{U}_{N}\right\|^{2}}
$$

where $\|\cdot\|$ denotes the Euclidean norm, $\mathbf{U}_{N}$ represents the noise subspace and comprises the eigenvectors of the covariance matrix $\mathbf{R}$ which are corresponding to the $M_{0}$ smallest eigenvalues, where $M_{0}$ is assumed to be known or can be estimated using the information theory methods $[9]$.

In practice, the covariance $\mathbf{R}$ needs to be updated and estimated from the average of samples

$$
\hat{\mathbf{R}}(n)=\frac{1}{K} \mathbf{V}_{\mathrm{cv}}(n) \mathbf{V}_{\mathrm{cv}}^{\mathrm{H}}(n)
$$

where

$$
\mathbf{V}_{\mathrm{cv}}(n)=\left[\begin{array}{ccc}
v_{\mathrm{cv}}(n) & \ldots & v_{\mathrm{cv}}(n-K+1) \\
\vdots & \ddots & \vdots \\
v_{\mathrm{cv}}(n-M+1) & \ldots & v_{\mathrm{cv}}(n-K-M+2)
\end{array}\right]
$$

and $K$ is the number of observations.

\subsection{Missing harmonic signals in the complex-valued signal}

In detail, $v_{\mathrm{cv}}(n)$ is composed of complex-domain harmonic signals that can be divided into two categories plus noise,

$$
v_{\mathrm{cv}}(n)=v_{\mathrm{cv} 1}(n)+v_{\mathrm{cv} 2}(n)+\varepsilon_{\mathrm{cv}}(n)
$$

where $v_{\mathrm{cv} 1}(n)$ is the summation of all positive sequence voltages,

$$
v_{\mathrm{cv} 1}(n)=\sum_{p=1}^{\left\lfloor\frac{H+2}{3}\right\rfloor} V_{3 p-2} \mathrm{e}^{i(3 p-2)\left(\Omega n T_{s}+\phi\right)}
$$

and $v_{\operatorname{cv} 2}(n)$ is the summation of all negative sequence voltages,

$$
v_{\mathrm{cv} 2}(n)=\sum_{p=1}^{\left\lfloor\frac{H+1}{3}\right\rfloor} V_{3 p-1} \mathrm{e}^{-i(3 p-1)\left(\Omega n T_{s}+\phi\right)}
$$

and $\lfloor x\rfloor$ denotes the largest integer not greater than $x$.

All the zero sequence voltages have been cancelled out. Zero sequence voltages of the same order are cophasial in the three voltage channels and will be eliminated since both rows of the transformation matrix $\mathbf{T}$ are zero-mean vectors.

To solve this problem, we propose our quaternionvalued approach in the next section.

\section{Quaternion-valued frequency estimation for three-phase power systems}

We construct a quaternion-valued signal from the three-phase signals as [7]

$$
v_{\mathrm{qv}}(n)=i v_{a}(n)+j v_{b}(n)+k v_{c}(n)
$$

where $i, j, k$ are the three imaginary units of the quaternion algebra which are constrained by $[8]$

$$
\begin{aligned}
& i^{2}=j^{2}=k^{2}=i j k=-1 \\
& i j=-j i=k \\
& j k=-k j=i \\
& k i=-i k=j
\end{aligned}
$$

This quaternion-valued signal contains quaternion-domain harmonic signals that belong to three categories $v_{\mathrm{qv} 1}(n), v_{\mathrm{qv} 2}(n), v_{\mathrm{qv} 3}(n)$ plus noise,

$$
\begin{aligned}
& v_{\mathrm{q}}(n)=i v_{a}(n)+j v_{b}(n)+k v_{c}(n)= \\
& v_{\mathrm{qv} 1}(n)+v_{\mathrm{qv} 2}(n)+v_{\mathrm{qv} 3}(n)+\varepsilon_{\mathrm{qv}}(n)
\end{aligned}
$$

where $v_{\mathrm{qv} 1}(n)$ is the summation of all the positive sequence voltages

$$
\begin{gathered}
v_{\mathrm{qv} 1}(n)=\sum_{p=1}^{\left\lfloor\frac{H+2}{3}\right\rfloor} \frac{2 i-j-k}{2} V_{3 p-2}\left\{\cos \left[(3 p-2)\left(\Omega n T_{s}+\phi\right)\right]\right. \\
\left.-\frac{i+j+k}{\sqrt{3}} \sin \left[(3 p-2)\left(\Omega n T_{s}+\phi\right)\right]\right\} \\
=\sum_{p=1}^{\left\lfloor\frac{H+2}{3}\right\rfloor} \frac{2 i-j-k}{2} V_{3 p-2} \mathrm{e}^{-\frac{i+j+k}{\sqrt{3}}(3 p-2)\left(\Omega n T_{s}+\phi\right)}
\end{gathered}
$$




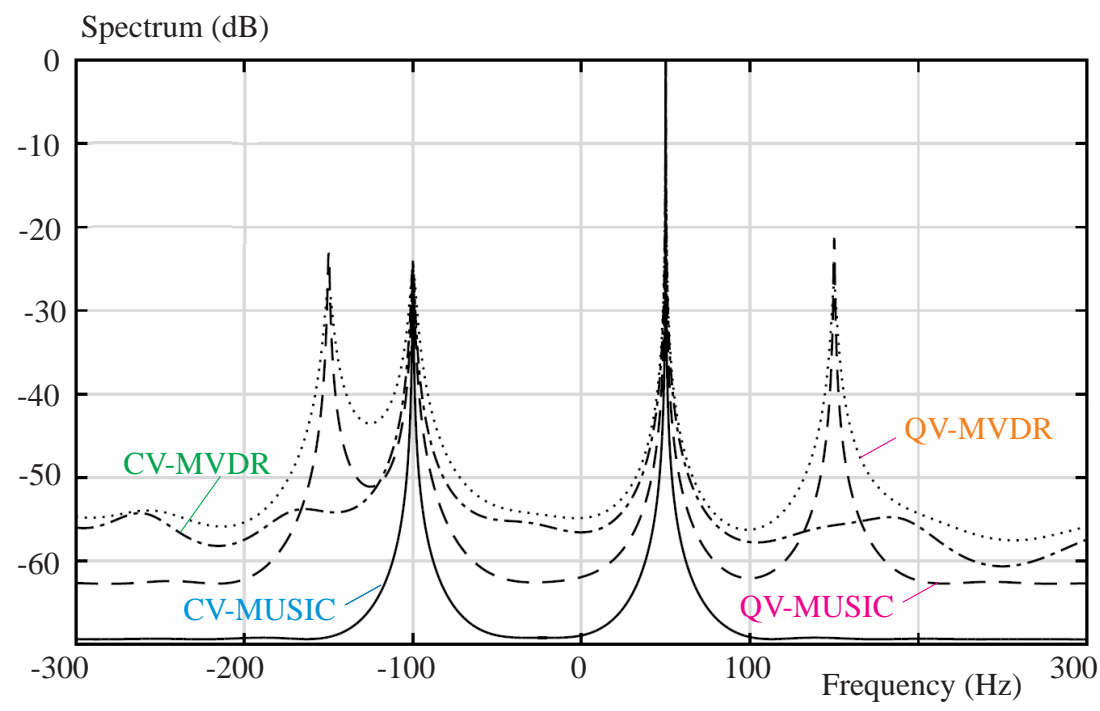

Fig. 1. MVDR and MUSIC spectra of quaternion-and complex-valued models

$v_{\text {qv2 }}(n)$ is the summation of all the negative sequence voltages

$$
\begin{gathered}
v_{\mathrm{qv} 2}(n)=\sum_{p=1}^{\left\lfloor\frac{H+1}{3}\right\rfloor} \frac{2 i-j-k}{2} V_{3 p-1}\left\{\cos \left[(3 p-1)\left(\Omega n T_{s}+\phi\right)\right]\right. \\
\left.+\frac{i+j+k}{\sqrt{3}} \sin \left[(3 p-1)\left(\Omega n T_{s}+\phi\right)\right]\right\} \\
=\sum_{p=1}^{\left\lfloor\frac{H+1}{3}\right\rfloor} \frac{2 i-j-k}{2} V_{3 p-1} \mathrm{e}^{\frac{i+j+k}{\sqrt{3}}(3 p-1)\left(\Omega n T_{s}+\phi\right)}
\end{gathered}
$$

and $v_{q v 3}(n)$ is the summation of all the zero sequence voltages

$$
\begin{array}{r}
v_{\mathrm{qv} 3}(n)=\sum_{p=1}^{\left\lfloor\frac{H}{3}\right\rfloor} V_{3 p}(i+j+k) \cos \left[3 p\left(\Omega n T_{s}+\phi\right)\right] \\
=\sum_{p=1}^{\left\lfloor\frac{H}{3}\right\rfloor} V_{3 p} \frac{i+j+k}{2}\left[\mathrm{e}^{\frac{i+j+k}{\sqrt{3}} 3 p\left(\Omega n T_{s}+\phi\right)}\right. \\
\left.+\mathrm{e}^{-\frac{i+j+k}{\sqrt{3}} 3 p\left(\Omega n T_{s}+\phi\right)}\right] .
\end{array}
$$

Hence all the harmonic signals will be reserved in the quaternion-valued signal. We may observe from (15)-(17) that the frequencies of the harmonic signals have been mapped into the frequencies of the quaternion-valued signal associated with the $\frac{i+j+k}{\sqrt{3}}$ axis.

The frequency estimation problem from a sum of quaternion-valued exponentials is similar to the problem in the complex domain. Then we can adopt the MVDR spectrum in (4) and the MUSIC spectrum in (6) by substituting the frequency sweeping vector as

$$
\mathbf{s}(\Omega)=\left[1, \mathrm{e}^{-\frac{1}{\sqrt{3}}(i+j+k) \Omega T_{s}}, \ldots, \mathrm{e}^{-\frac{M-1}{\sqrt{3}}(i+j+k) \Omega T_{s}}\right]^{\top} .
$$

NOTE-1: Not to be confused with the Quaternion-MVDR (Q-MVDR) algorithm [10] for the adaptive beamforming with vector-sensor array beamforming or the QuaternionMUSIC (Q-MUSIC) algorithm [11] for the direction-of-arrival estimation with vector-sensor arrays. Their steering vectors are complex-valued vectors multiplied by quaternionvalued scalars, which are conceptionally different from the quaternion-valued frequency sweeping vector defined in this paper. We marked our algorithms by QV-MVDR and QVMUSIC for clarification.

The frequencies detected in the spectrum are either the original real-domain angular frequencies or their additive inverses, namely

(1) If a peak is detected in the spectrum in the absence of its additive inverse, it corresponds to a positive or negative sequence voltage signal and this spectrum peak indicates its angular frequency or its additive inverse.

(2) If two "mirrored" peaks are detected in the spectrum, they correspond to a zero sequence voltage signal and they indicate the signal's angular frequency and its additive inverse, respectively.

\section{Simulations}

In this section, we provide a numerical example to illustrate the performance of the proposed quaternion model. The fundamental frequency is $50 \mathrm{~Hz}$, the sampling frequency is $f_{s}=1 / T_{s}=20 \mathrm{kHz}$, the initial phase is $\phi=\pi / 7$, and $K=80, M=32$. There exist a secondorder and a third-order harmonic signals, both set to be $6 \%$ in amplitude.

We test the capability of the two modelings. The MVDR and MUSIC spectra of the quaternion- and complex-valued models are plotted in Fig. 1, where $\mathrm{SNR}=40 \mathrm{~dB}$. It can be observed that the proposed model is able to detect all the harmonic signals, namely 
$50 \mathrm{~Hz}$ (the fundamental frequency), $-100 \mathrm{~Hz}$ (the secondorder harmonic), and $\pm 150 \mathrm{~Hz}$ (the third-order harmonic), while the complex-valued model fails at the thirdorder harmonic frequency.

\section{Conclusion}

We have presented a quaternion-valued model as an alternative preprocessing approach to convert the threephase signals into a single-phase system. Compared with the Clarke's transformation, the proposed model can additionally detect the zero sequence voltages. Simulated results show that the proposed model can detect all-order voltage harmonics effectively.

\section{Acknowledgements}

This work was supported by the National Natural Science Foundation of China (Grant Nos. 61331019 and 61490691).

\section{REFERENCES}

[1] M. Bollen, "Understanding Power Quality Problems: Voltage Sags and Interruptions", Wiley-IEEE Press, 2000.

[2] M. Akke, "Frequency Estimation by Demodulation of Two Complex Signals", IEEE Transactions on Power Delivery, vol. 12, no. 1, pp. 157-163, Jan 1997.

[3] H. J. Jeon and T. G. Chang, "Iterative Frequency Estimation based on MVDR Spectrum", IEEE Transactions on Power Delivery, vol. 25, no. 2, pp. 621-630, Apr 2010.

[4] Y. Xia and D. P. Mandic, "Augmented MVDR Spectrum-Based Frequency Estimation for Unbalanced Power Systems", IEEE Transactions on Instrumentation and Measurement, vol. 62, no. 7, pp. 1917-1926, July 2013.

[5] R. O. Schmidt, "Multiple Emitter Location and Signal Parameter Estimation", IEEE Transactions on Antennas and Propagation, vol. 34, no. 3, pp. 276-280, Mar 1986.

[6] W. M. Grady and S. Santoso, "Understanding Power System Harmonics", IEEE Power Engineering Review, vol. 12, no. 11, pp. 8-11, Nov 2001.

[7] S. P. Tabeli and D. P. Mandic, "A quaternion Frequency Estimator for Three-Phase Power System", Proceedings of 2015 IEEE International Conference on Acoustics, Speech and Signal Processing (ICASSP), South Brisbane, Queensland, Australia, pp. 3956-3960, Apr 2015.

[8] J. P. Ward , Quaternions and Cayley Numbers, Algebra and Applications", Kluwer, Normwell, MA, 1997.

[9] M. Wax and T. Kailath, "Detection of Signals by Information Theoretic Criteria", IEEE Transactions on Acoustics, Speech, and Signal Processing, vol. ASSP-33, no. 2, pp. 387-392, Apr 1985.

[10] J. W. Tao, "Performance Analysis for Interference and Noise Canceller based on Hypercomplex and Spatio-Temporal-Polarisation Processes", IET Radar Sonar and Navigation, vol. 7, no. 3, pp. 277-286, Mar 2013.
11] S. Miron, N. Le Bihan and J. I. Mars, "Quaternion-MUSIC for Vector-Sensor Array Processing", IEEE Transactions on Signal Processing, vol. 54, no. 4, pp. 1218-1229, Apr 2006.

Received 19 October 2017

Xiaoming Gou received the $\mathrm{BS}$ and $\mathrm{PhD}$ degrees from Beijing Institute of Technology, China, in 2010 and 2016, respectively, all in electronic engineering. His main research interest is array signal processing within the hypercomplex framework.

Yougen $\mathrm{Xu}$ received the MS and PhD degrees from Beijing Institute of Technology, China, in 2001 and 2004, respectively, all in electronic engineering. In September 1998, he joined the Institute of Signal and Image Processing, Beijing Institute of Technology, where he has been involved in projects related to direction finding, digital adaptive robust beam-forming and blind source extraction with advanced vector sensors. Since 2004, he has been with the School of Information and Electronics, Beijing Institute of Technology, where he is currently a Professor. His research interests are mainly in the area of array signal processing, wireless sensor network, regularization methods and applications in sensor array signal processing, biomedical digital signal processing and space-time adaptive processing. He is a member of the Chinese Institute of Electronics.

Zhiwen Liu received the BS degree from Xidian University, Xi'an, China in 1983, and the MS and PhD degrees from Beijing Institute of Technology, Beijing, China in 1986 and 1989, respectively, all in electronic engineering. Since 1989, he has been with the School of Information and Electronics, Beijing Institute of Technology, where he is currently a Professor. His research interests include radar imaging, detection and estimation theory, video image processing, array signal processing, with applications in communications, radar and life informatics. He is the coeditor of the 1996 CIE International Conference of Radar Proceedings. He received the 1995 Distinguished Younger Teacher Award from the Beijing Municipal Government, and won the Higher Education Teaching Award from the Beijing Municipal Government in 2001. He is a senior member of the Chinese Institute of Electronics.

Wei Liu received his BSc in Space Physics in 1996, LLB in Intellectual Property Law in 1997, both from Peking University, China, MPhil from the Department of Electrical and Electronic Engineering, University of Hong Kong, in 2001, and $\mathrm{PhD}$ in 2003 from the School of Electronics and Computer Science, University of Southampton, UK. He then worked as a postdoc in the same group and later in the Communications and Signal Processing Group, Department of Electrical and Electronic Engineer-ing, Imperial College London. In September 2005, he joined the University of Sheffield, UK, as a Lecturer and now he is a Senior Lecturer. His research interests are mainly in sensor array signal processing, blind signal processing, multirate signal processing and their various applications such as wire-less communications, sonar, radar, satellite navigation, and speech enhancement, etc. 